\title{
热带、亚热带玉米育种素材的遗传改良
}

\author{
三森・健菢䑣, 查芭・健菢䑣
}

泰国肯色萨大学, 国家玉米和高梁研究中心/国家苏湾农场, 帕创 那苛拉查西马 30320

摘要: 以泰国玉米品种与适应种质共 122 份为素材, 采用改良穗行、顶交或测交方案, 按精准的育种目标与定 向设计, 创制出具有鲜明独特特征的开放授粉品种(Open pollinate varieties, OPVs)。育种素材选择考虑表现优 良、广适性、遗传多样性等 3 个因素; 合成系统考虑实现遗传混合与重组、均等比较遗传贡献、施之温和选择 压力、充分交配达到遗传平衡(遗传组成母本性状相近)等 4 条要素。相继创制 Suwan 1 复合种及其(半)硬粒型 衍生种(Suwan 2、3 复合种, Suwan 5、KS24 综合种), 含有不同血缘的马齿型 KS6、KS28, Non-Suwan 1 血缘的 马齿型 Caripeno DMR、KS27、KS23 等。对 10 个 OPVs 采用 S1 选择法分别实施了 C2 C13 轮选择, 从中育 成近 50 个自交系, 组配并发放 16 个优良单(三)交种。目前泰国各单位育成的每个杂交种中, 至少有一个亲本 系来源于 Suwan(KS)种质。文章还依据合成 OPVs 及选系理论, 对合成 OPVs 遗传成分适度多样化、亲缘关系、 优势群与模式和合成 OPVs 对杂交优势育种的启示问题加以探讨。

关键词: 玉米 OPVs; 硬粒与马齿型 OPVs 合成; 单(三)交种; 遗传变异性与多样性

\section{Genetic improvement of breeding materials in tropical and sub- tropical maize}

\section{Sansern Jampatong, Chaba Jampatong}

National Corn and Sorghum Research Center / Farm Suwan, Kasetsart University, Pakchong, Nakhon Ratchasima 30320, Thailand

\begin{abstract}
In the present study, 122 maize local cultivars and adapted exotic germplasm from Thailand were used to develop open pollinate varieties (OPVs) using modified ear-to-row scheme, top-cross or test-cross programmes. Ten new maize OPVs with distinct characters were created based on the precise breeding objectives and directional design. The selection of breeding materials was based upon three factors: elite performance, broad adaptability, and genetic diversity. The synthesizing system provided four features: genetic mixing and recombination, equal comparable genetic contribution, mild selection pressure, and maximum intermating for genetic equilibrium (i.e., the female traits were close for the genetic compositions). Subsequently, Suwan 1 composite and its deritives (Suwan 2, Suwan 3 composite, Suwan 5 and KS24 synthetics), KS6 and KS28 synthetics with the dent type of different origins, and Caripeno DMR composite, KS23, and KS27 synthetics with the dent type of Non-Suwan 1 origin were developed. These OPVs had been improved for 2 13 cycles using S1 recurrent selection method. About 50 inbred lines were developed from these OPVs, and 16 elite single (three-way) crosses were combined and released from these inbred lines. At present, at least one parental inbred line of all the tropical hybrids
\end{abstract}

收稿日期: 2010-11-11; 修回日期: 2011-10-17

作者简介: 三森· 健萉粀, 博士, 专家研究员, 研究方向 : 玉米遗传改良、种质创新。E-mail: snjpt@loxinfo.co.th

致谢: 中国科学院遗传与发育生物学研究所曾孟潜研究员对本文的编译及修改付出了大量的时间和精力，作者在此表示哀心的感谢 ! 
was derived from Suwan (KS) germplasm in Thailand. Based on the theory of the synthesizing OPVs and developing inbred lines, this paper discussed the genetic moderate diversity, relationship, heterotic group, and patterns for synthesizing OPVs, and inspiration for composed OPVs to heterosis breeding.

Keywords: maize OPVs; composed OPVs of flint and dent types; single, three-way cross; genetic variability and diversity

玉米引入泰国已有相当长的历史, 1932 年开始 规模化商品生产, 引入 Nicholson YD 与 Mexican June 等品种, 1953 年引入“ Guatemala” (Tequisate GYF) 等优良品种 ${ }^{[1,2]}$ 。1958 1959 年肯色萨大学 (Kasetsart University, KU)开始玉米遗传改良的研究, $\mathrm{KU}$ 于 1965 年设立国家玉米和高梁研究中心(National Corn and Sorghum Research Center, NCSRC)/苏湾农 场(Farm Suwan, FS), 1963 年农业部农业厅(Department of Agriculture, Ministry of Agriculture. DOA MA)所属全国大田作物研究所 (National Field Crops Institute, NFCI) 设立那苛沙湾大田作物研究中心 (Nakhon Sawan Field Crops Research Center, NSFCRC) 等两个从事玉米科研的国有专业机构。1966 年国际 亚洲玉米项目(IACP)总部迁移到 FS(Farm Suwan), 促成大规模引进育种素材, 从国际玉米和小麦改良 中心(International Maize and Wheat Improvement Center, CIMMYT)引进来自加勒比地区、墨西哥、印 度的大批优良 OPVs, 从菲律宾、印尼、台湾地区引 进大量复合种(Composite, Comp)与其他热带、亚热 带种质, 建设种质库, 长期保存并利用这批材料。通 过种植、观察、研究、改良, 篮选到以来源于加勒 比地区为主的一批优良育种素材 ${ }^{[1 \sim 4]}$ 。1971 1974 年, 高梁霜霉病(Sorghum downy mildew, DM)在泰国大 规模发生, Tequisate GYF 停止生产, 转而种植 Tainan No.10 与 Bogore 综合种(Synthetics, Syn)和 KU 选育的 Thai DMR No.6 OPV。由于品种遗传多 样性的限制, 未能配制出强优势的品杂, KU 团队与 CIMMYT 团队通力协作集中力量开展 OPVs 选育 ${ }^{[3,4]}$ 。 分别于 1969 1972 年、1969 1974 年合成 Suwan 1 和 Suwan 2, 又从世界各地引进大批育种素材, 合成 近 50 个基础群体(1969 2005), 并分别作了 C2 C13 轮选择, 选出近 50 个优良系(1982 2008), 组配出 16 个优良单(三)交种, 大规模应用于生产 ${ }^{[2,4]}$ 。杂交玉
米研究始于 1978 年(KU), 配制成的 SW2301 第一个 单交种于 1982 年在泰国首次种植。1978 1982 年间 又一次从热带、亚热带及温带地区引进大批育种素 材, 篮选配合力、农艺性状优良材料, 参与合成 Suwan 1 衍生 Comp, Syn 及与它相对应的相互轮选 Syn 基础群体 KS23。单(三)交种生产基本普及后, 国 有与私营玉米机构开展科研合作与信息交流, 也有 力地推动育种和生产的发展。

本文阐述了泰国玉米育种、生产, 从开放授粉 品种(Open pollinate varieties, OPVs)发展到单(三)交 种的历程, 首次全面发表 NCSRC/FS KU 玉米遗传 育种定向设计、应用的育种素材与方法、取得的成 果(就), 并对育种素材改良一些热点问题加以讨论, 为今后进一步开展育种素材改良研究提供实践参 照、借鉴和科学依据。

\section{1 材料和方法}

制定的育种目标：高产, 稳产, 优质, 优良直立 性, 适宜机械化, 耐密性好, 多抗, 抗耐非生物胁迫, 具广适性、中晚熟、籽粒橙黄、属硬、半硬粒型或 半马齿型, 能经济利用水分养分资源。母本系强调 产种量要高, 父本系花药开裂性能要好, 能散发足 够花粉。要求易于繁殖, 繁殖系数高, 制种操作简单, 快捷。定向设计：首先育成标准 OPV, 然后创制其 衍生 OPVs, 配套使用, 形成丰富的遗传基础、遗传 多样性、变异性; 进一步搜集与创新育种素材及其 他种质, 通过配合力测定与分析, 合成与标准 OPV 配对的轮选 Syn 基础群体。而后用系谱法从轮选群 体与优良组合选育自交系, 组配单(三)交种。

\section{1 材料}

对参与新轮选群体的遗传组成一育种素材的选 择应具备 3 个因素: 表现优良(秀)、广适性、遗传多 
样性 ${ }^{[4]}$ 。研究应用试材见表 1 。

\section{2 方法}

应用了 3 种方法 :(1)改良的穗行方案 ${ }^{[1]}$; (2)顶交 方案: 选定测验品种与遗传组成各方直接做顶交, 或遗传组成各方每方提取 10 15个 S1 系后, 再做顶 交; (3)测交方案：选定测验系与遗传组成各方做测 交。应用 3 种方法共同点为：遗传组成各方均经一 般、特殊配合力测定与分析; 杂交顶(测)交、互交植 株数量较大, 选择压力 (中选率)不太大; 遗传组成 泰国品种较少, 外来优秀的适应种质较多。合成 Comp, Syn 必须达到“ 遗传平衡” 要求, 判别遗传平 衡的一个重要元素是:小区间多父本授粉的各个遗 传组成母本一些特征特性上可观察到细微差异, 而 总体上看所有母本植株(果穗、籽粒)性状很相近。无 论应用哪些种质, 哪种方法合成, 都要求达到 4 条 要素: 实现系统的遗传混合与重组、均等比较遗传 贡献、充分的交配、施之以温和的选择压力 ${ }^{[1]}$ 。以 创制遗传基础丰富、有足够遗传变异性、类群清晰, 对育种有重要价值的新育种素材。10个 Comp, Syn 合成期间，均经过暴风雨、高密度(83333 株/ha.)、耐 旱、耐痊考验, 诱发与人工接种病菌的鉴定、选择。

10 个 Comp, Syn 合成后, 均用 S1 选择法进行轮 选, 重点改良籽粒产量、株高、抗倒性、熟期和配 合力。(半)硬粒类型 Comp, Syn 中, Suwan 1 进行了 C13 轮选, Suwan 2 为 C7, Suwan 3、Suwan 5、KS24 分别为 C7、C5、C3; 马齿型 Comp, Syn、KS23、 KS6、Caripeno DMR、KS27 与 KS28 则分别为 C6、 $\mathrm{C} 4 、 \mathrm{C} 8 、 \mathrm{C} 3$ 与 $\mathrm{C} 2$ 。C 1 轮后不同轮次的轮选均与系 谱法选系相结合进行。

\section{2 结果与分析}

\section{1 合成复合(综合)种主要特征、特性}

Suwan 1 复合种：硬粒型、中晚熟种, 见图 1。 平均株高 $253 \mathrm{~cm}$, 穗位 $111 \mathrm{~cm}$, 穗长 $22.6 \mathrm{~cm}$, 穗行 数 12 16, 单株粒重 $153 \mathrm{~g}$, 出籽率 $84.5 \%$, 千粒重 $310 \mathrm{~g}$ 。叶鞘紫色, 幼苗较整齐, 叶片大小中等, 株形 较紧凑。穗形长雉, 个别筒形, 穗轴白色, 籽粒橙黄, 个别黄粒。高抗倒伏(折)、抗霜霉病, 中抗或耐南方 锈病、穗腐病与小斑、大斑病, 耐旱涝, 贫痟胁迫,
从低轮次至高轮次生活力、发苗势、株高、品质、 果穗性状不断改善。(s)C10 较之(s)C4 自身籽粒产量 增高 15\%, 较 CK(Tequisate GYF)增产 17\% 30\%。20 世纪 80 年代前种植面积曾占到全国玉米总面积的 $80 \%$ 以上。

Suwan 2 复合种 : (半)硬粒型、中早熟种。平均 株高 $240 \mathrm{~cm}$, 穗位 $99 \mathrm{~cm}$, 穗长 $21.7 \mathrm{~cm}$, 穗行数 12 16, 单株粒重 $137 \mathrm{~g}$, 出籽率 84.6\%, 千粒重 293 g。与 Suwan 1 比较, 遗传成份相同; 各性状中除早 熟 $7 \mathrm{~d}$ 外, 大体上也相似(未提供照片)。广泛用于笋 玉米的采收与籽粒收获兼用的玉米生产。

Suwan 3 复合种: (半)硬粒型、中熟种, 见图 1。 平均株高 $257 \mathrm{~cm}$, 穗位 $115 \mathrm{~cm}$, 穗长 $23.7 \mathrm{~cm}$, 穗行 数 12, 单株粒重 $176 \mathrm{~g}$, 出籽率 $85.0 \%$, 千粒重 312 g。叶鞘紫色, 叶片较大, 株形较紧凑, 保绿性较好, 果穗长雉-筒形, 黄粒。配合力较高。抗倒、抗霜霉、 南方锈病、穗腐病、小斑病, 高抗茎腐。抗逆性与 果穗性状较同轮次的 Suwan 1 好, 自身籽粒产量较 Suwan 1 同轮次的增产 $15 \%$ 。

Suwan 5 综合种：硬粒型、中晚熟种, 见图 1。 平均株高 $260 \mathrm{~cm}$, 穗位 $113 \mathrm{~cm}$, 穗长 $24.1 \mathrm{~cm}$, 穗行 数 12 14, 单株粒重 $167 \mathrm{~g}$, 出籽率 $85.3 \%$, 千粒重 $317 \mathrm{~g}$ 。叶鞘浅紫色, 叶片较大, 株形较紧凑, 较繁茂, 保绿性好, 果穗筒形, 黄粒。配合力高, 籽粒产量较 Suwan 3 同轮次的增产 $12 \%$ ，被推荐为青饲青咜用 标准综合种。

KS24 综合种：(半)硬粒型、中晚熟种, 见图 1。 平均株高 $250 \mathrm{~cm}$, 穗位 $108 \mathrm{~cm}$, 穗长 $22.5 \mathrm{~cm}$, 穗行 数 12 , 单株粒重 $158 \mathrm{~g}$, 出籽率 $85.5 \%$, 千粒重 314 g。叶鞘紫色, 幼苗整齐, 叶片中等大小, 株形较紧 凑，根系发达，穗形筒形，黄粒。配合力比 Suwan 1 显著提高, 与 Suwan 1 比较, 在生活力、发苗势、抗 病抗逆、雄穗大小、果穗大小、籽粒产量上更好些。

KS23 综合种：马齿型、中晚熟种。见图 1 。 平均株高 $241 \mathrm{~cm}$, 穗位 $110 \mathrm{~cm}$, 穗长 $23.1 \mathrm{~cm}$, 穗行 数 12 14, 单株粒重 $155 \mathrm{~g}$, 出籽率 $86.1 \%$, 千粒重 $315 \mathrm{~g}$ 。叶鞘紫红色, 生长较整齐, 根系发达, 叶片较 窄, 较直立, 雄穗主轴、分枝分明, 穗形偏筒形, 粒 色橙黄与黄粒果穗掺合。配合力较 KS6 提高明显, 抗倒, 高抗霜霉、茎腐、南方锈病, 兼抗其他病害。 


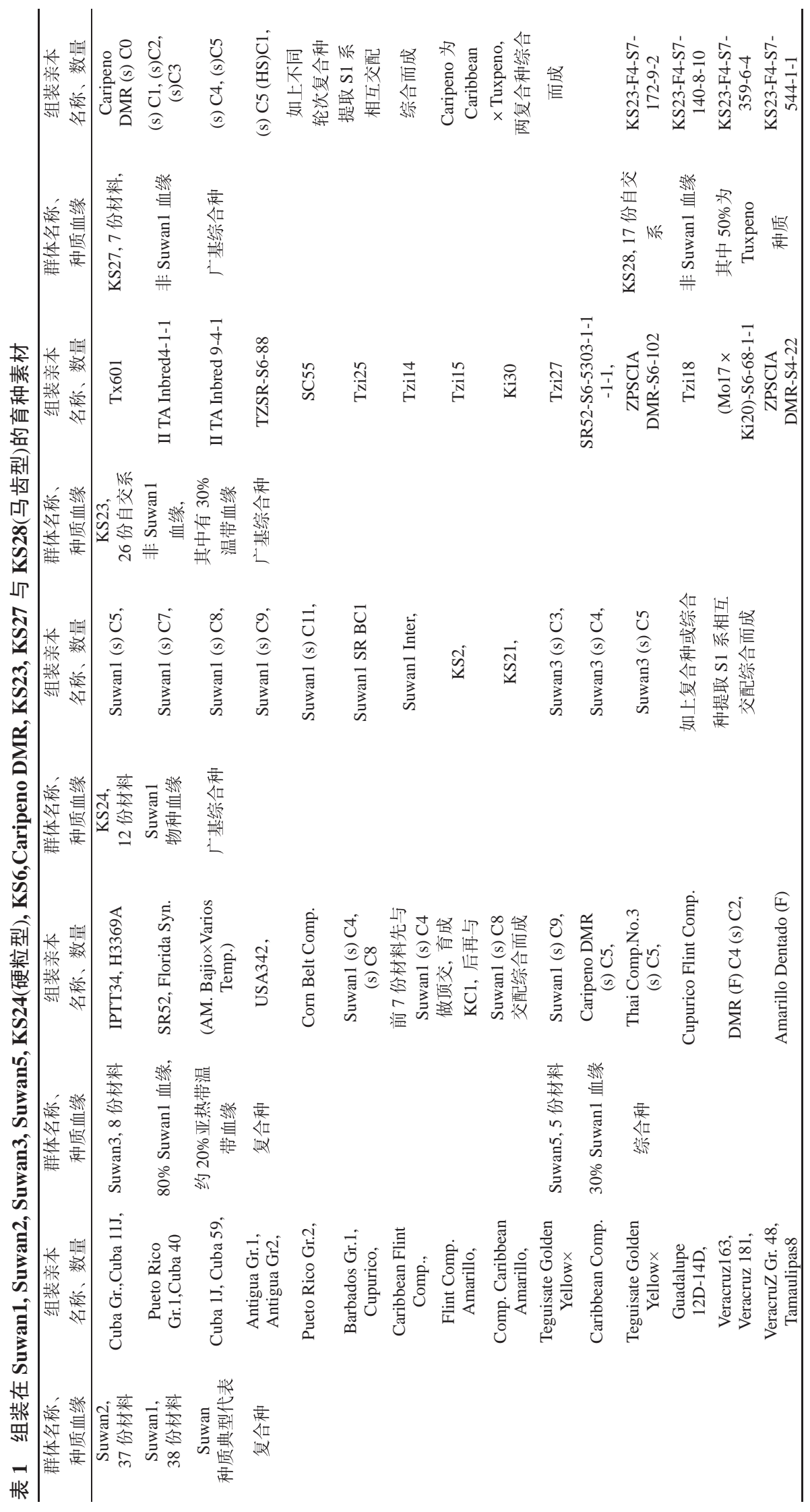




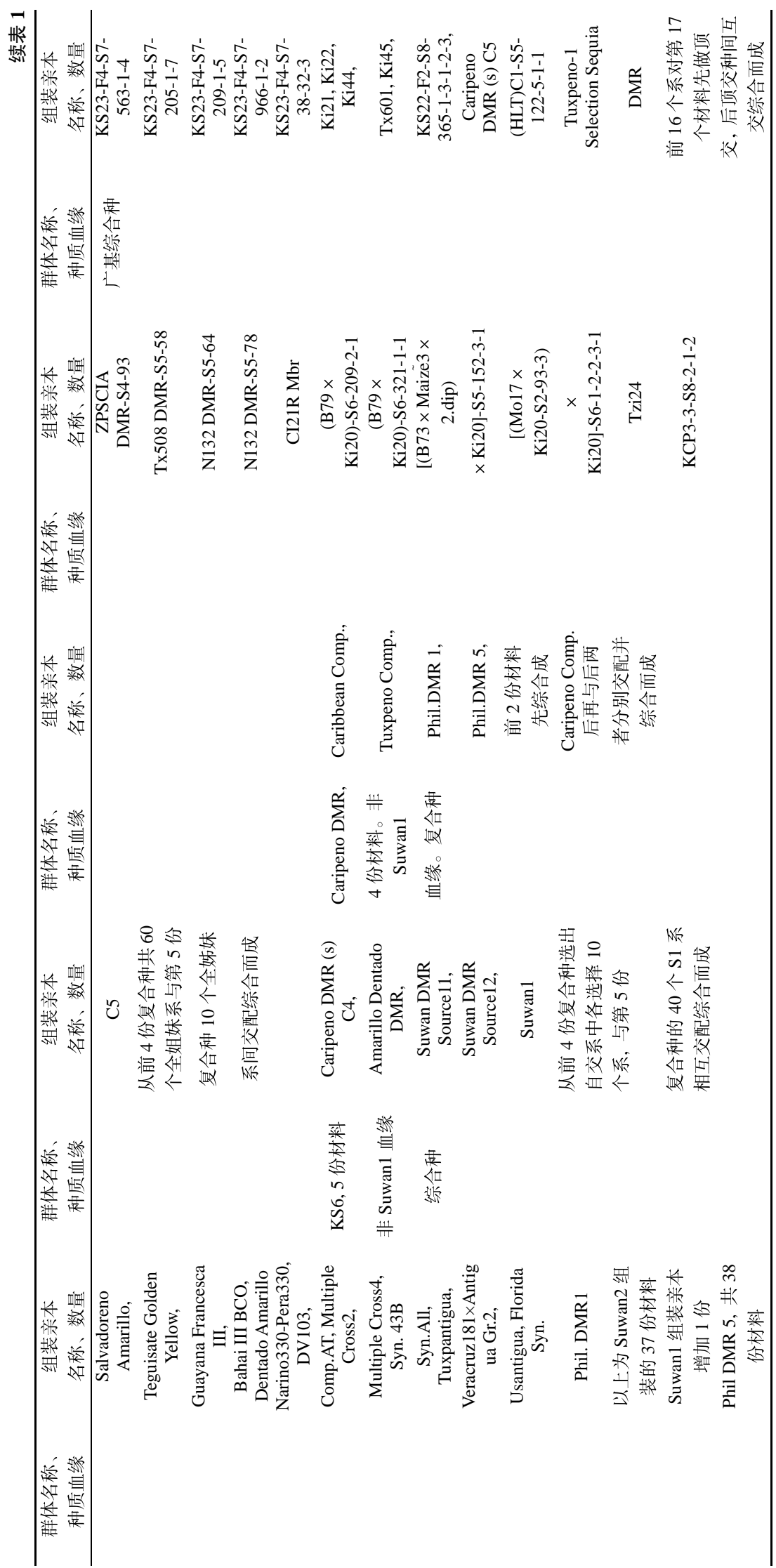



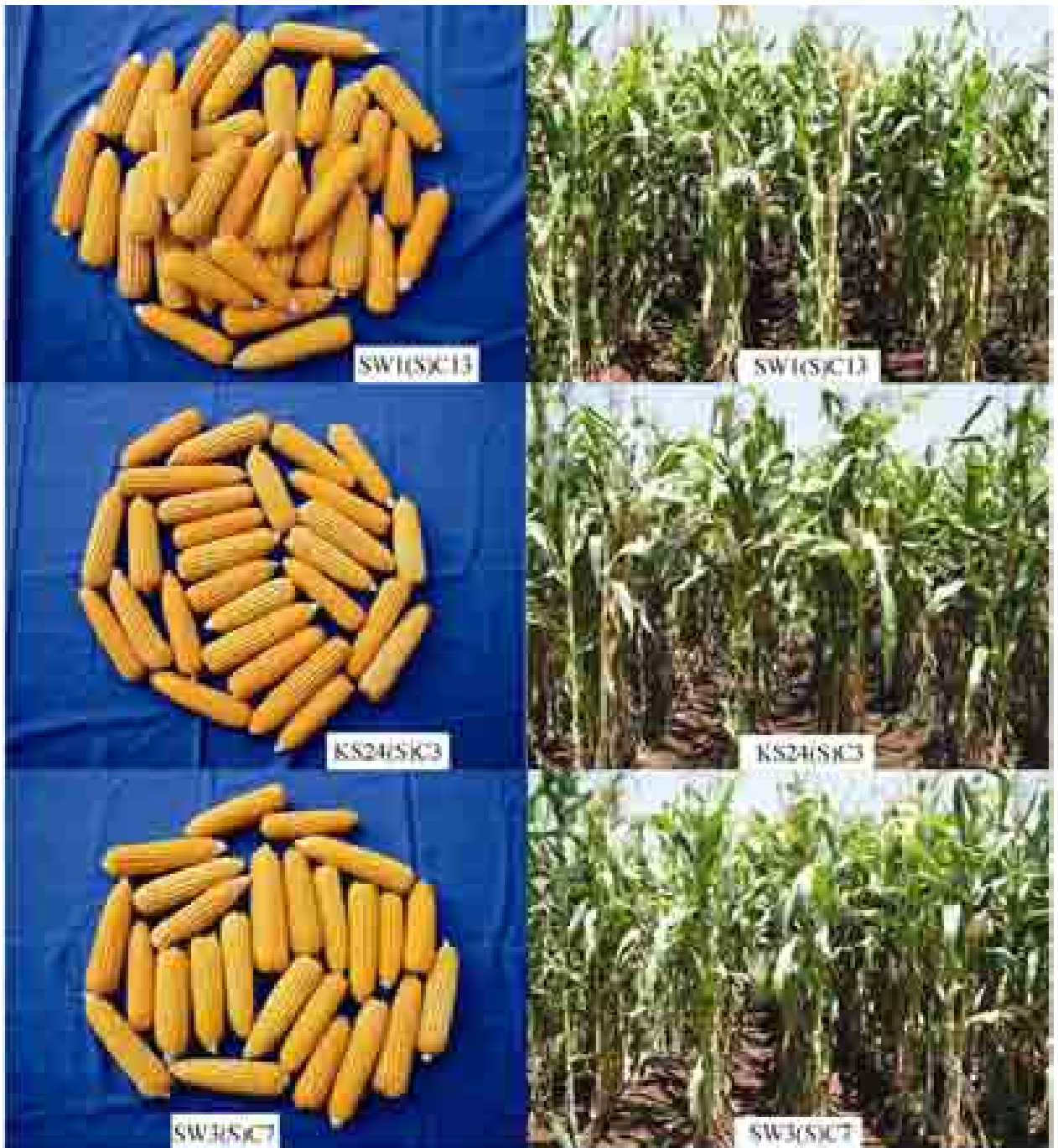

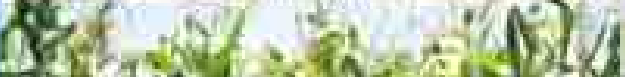

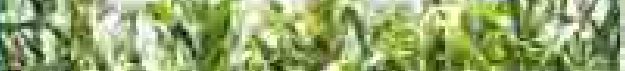

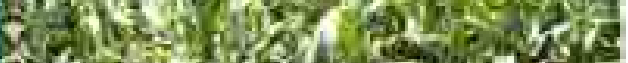

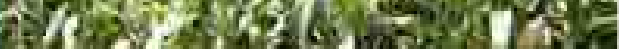

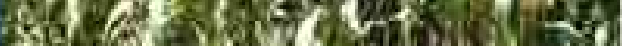

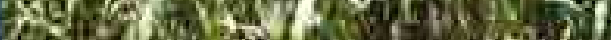

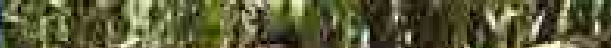

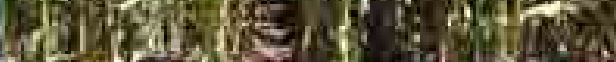
if

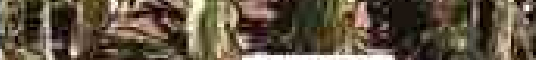

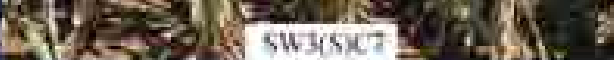

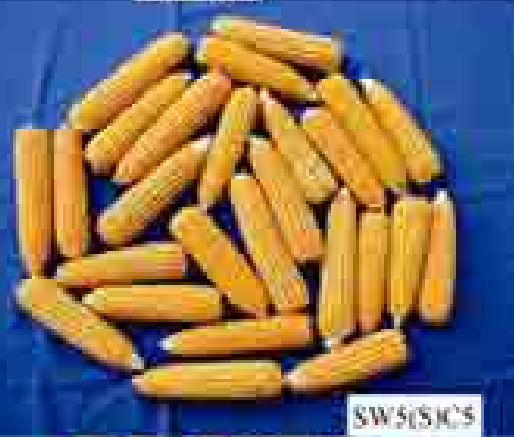

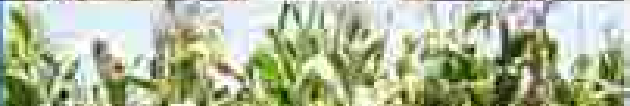

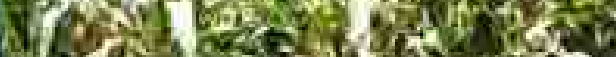

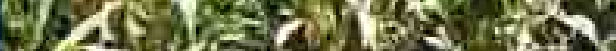
of Qfing

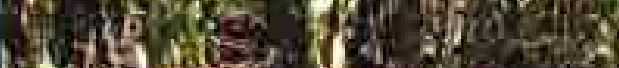
Q.

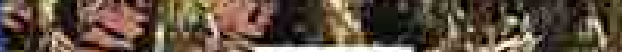

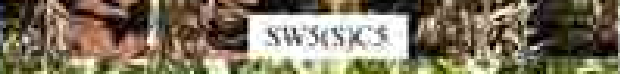
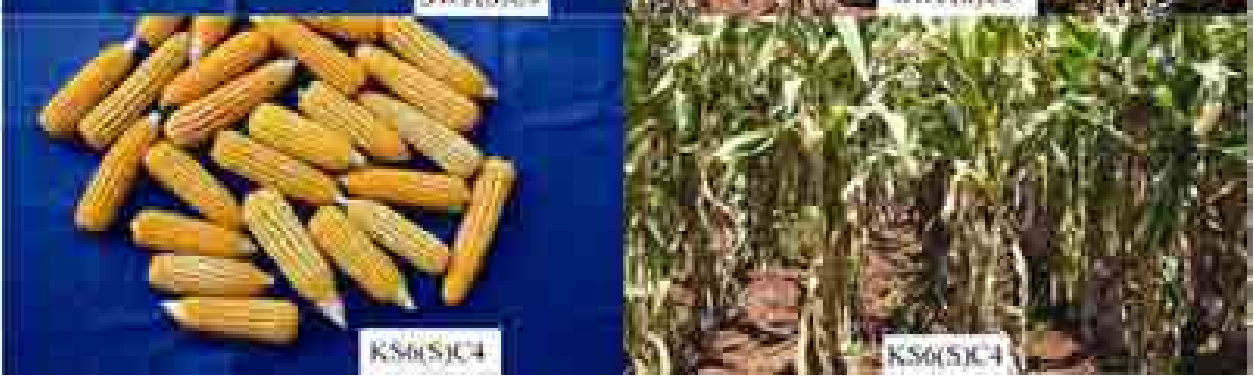


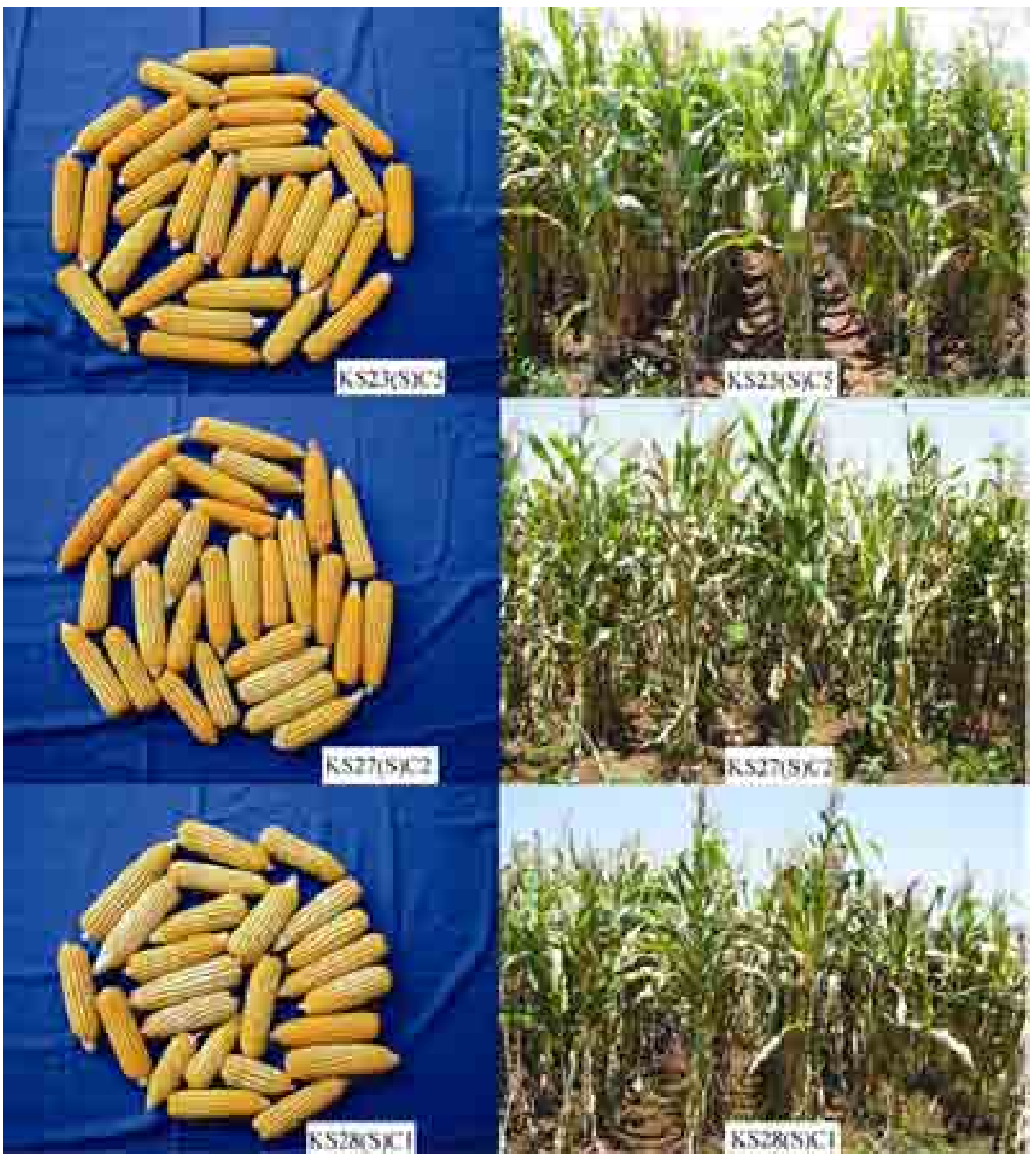

图 1 合成复合(综合)种主要特征图

KS6 综合种：马齿型、晚熟。见图 1。平均株 高 $265 \mathrm{~cm}$, 穗位 $121 \mathrm{~cm}$, 穗长 $24.0 \mathrm{~cm}$, 穗行数 12 14, 单株粒重 $168 \mathrm{~g}$, 出籽率 85.4\%, 千粒重 311 g。叶鞘紫色, 叶片中等大小, 株形一般, 雄穗分枝 多、发达, 粒色不整齐, 橙黄粒与黄粒穗掺合。抗霜 霉、茎腐病, 高抗南方锈、穗腐病, 抗逆性强。

Caripeno DMR 复合种：马齿型、晚熟种。平 均株高 $255 \mathrm{~cm}$, 穗位 $115 \mathrm{~cm}$, 穗长 $22.0 \mathrm{~cm}$, 穗行数 12 14, 单株粒重 $151 \mathrm{~g}$, 出籽率 $85.5 \%$, 千粒重 310 g。高抗霜霉、茎腐病, 兼抗小斑、大斑病等(未提供 照片)。

KS27 综合种：马齿型、晚熟种, 见图 1。平均 株高 $249 \mathrm{~cm}$, 穗位 $112 \mathrm{~cm}$, 穗长 $22.3 \mathrm{~cm}$, 穗行数 12 ,
单株粒重 $149 \mathrm{~g}$, 出籽率 $85.2 \%$, 千粒重 $310 \mathrm{~g}$ 。粒色 不整齐, 橙黄粒与黄粒穗掺合, 穗形长雉形, 配合 力较 Caripeno DMR 明显提高。高抗倒、抗霜霉、南 方锈、茎腐病, 耐小斑、大斑病等。

KS28 综合种：马齿型、晚熟种, 见图 1。平均 株高 $247 \mathrm{~cm}$, 穗位 $120 \mathrm{~cm}$, 穗长 $22.0 \mathrm{~cm}$, 穗行数 12 14, 单株粒重 $154 \mathrm{~g}$, 出籽率 $85.4 \%$, 千粒重 313 g。叶片较大, 株形一般, 粒色以黄粒为主, 少数橙 黄粒。配合力较 KS6、KS27 明显提高。抗倒性强、 高抗霜霉病, 耐茎腐、南方锈病, 兼抗小斑、大斑病等。

\section{2 合成复合(综合)种遗传组成}

合成的 4 个复合种与 6 个综合种是按制定的育 种目标与定向设计及具体掌握 3 个因素精细选择合 
成的。其遗传组成见表 1 。

Suwan 1: 共 38 份材料, 用改良的穗行方案(包 括回交法 $)^{[1]}$ 合成。合成经过 4 次交配, 第一季收获 $50 \%$ 母本果穗，第二、三、四季分别收获 $36 \%$ 、30\%、 少于 $30 \%$ 母本果穗。第四季后达到“遗传平衡”, 命 名为 Thai Comp No. 1 CO, 经用 S1 选择法施行一轮 轮选, 经过暴风雨考验, 选择 700-1000S1 家系, 选 择压力 $7 \% \sim 10 \%$, 得到 Thai Comp No. 1 C1 群体, 又与抗 DM、丰产适应外来种质 Phil(Philippine, Phil)DMR 1 与 5 杂交, 再以抗霜霉病种质回交 3 次 (BC3), 最后获得 Thai Comp 1 DMR BC3(s) C2, 定 名为 Suwan 1; Suwan 2 :共 37 份材料, 从 Thai Comp No.1(s)C1 中选择早熟 $7 \mathrm{~d}$ 的母本植株果穗, 再用 Phi1 DMR 1 与之杂交、回交, 后经过 S1 选择法轮选 至 C4, 即 Thai Comp No.1 Early(s)C4, 定名为 Suwam 2; Suwan 3 : 共 8 份材料, 用顶交方案合成; Suwan 5 : 用 7 份材料的 S1 系(或部分自交系), 以测 交方案合成; Caripeno DMR, KS6, KS24, KS23、 KS27 和 KS28 分别用 4 份、5 份、12 份、26 份、 7 份、17 份材料, 以测交方案合成。每个 Comp, Syn 均须系统考虑 4 条要素, 达到遗传平衡要求 ${ }^{[1,4]}$ 。

\section{3 杂交优势育种及其在生产上的应用}

从合成 10 个 Comp,Syn 选育出的主要自交系及 其应用见表 2 。由表 2 可知, 从 2 个单交种 $F_{2}$ 群体 Pac. 9、Pn3013 分别选育和配成 2、7 个与 $1 、 6$ 个
自交系、杂交种(图 2); Suwan 1,(s)C4,(s)C6,(s)C7, (s)C8,(s)C9,(s)C10(HLT)C1-F2 轮选育成 32 个系配成 6 个杂交种(图 3); Suwan 2,(s)C7 选育成 1 个系(Ki40); Caripeno DMR, (s)C1 选育成 2 个系配成 1 个杂交种 (图 3); Suwan 3, (s)C1,(s)C2,(s)C3 轮选育成 5 个系配 成 3 个杂交种(图 3); Suwan 5, 选育成 1 个系配成 1 个杂交种(KSX4802); KS6,(s)C2 选育成 2 个系配成 4 个杂交种(图 3); 从 KS23-F4 选育成 4 个系配成 1 个 杂交种(KSX4803)。KS24、KS27、KS28 则尚未选育 出自交系。据估计 ${ }^{[2,3]}$, 目前在泰国各个育种研究机 构所育成的每个杂交(单交、改良单交、三交)种中, 至少有 1 个亲本系选自 Suwan(KS)种质。Suwan 1 是标准 Comp, Suwan(KS)种质典型代表, Suwan 2 具 有 $100 \%$ 的 Suwan 1 血缘; Suwan 3、5、KS24 与上述 2 个 Comp 同为硬粒型 Comp 或 Syn, Suwan 3 具有 80\%的 Suwan 1 血缘及 20\%亚热带温带玉米血缘。 Suwan 5 具有 32\%的 Suwan 1 血缘、22\%的 Caripeno DMR、46\%的 Non- Suwan 1 血缘; KS24 具有近 100\% 的 Suwan 1 血缘。属于马齿型的 5 个 Comp,Syn, 均 为 Non-Suwan 1 血缘。但 Caripeno DMR 遗传组成都 参与合成 Suwan 1, KS27 与 Caripeno DMR 血缘相 同; KS6 遗传成分中有 3 份与 Suwan 1 的相同; KS28 具有 $50 \%$ 为 Tuxpeno 血缘, 遗传成分中仍有 3 份曾 参与合成 Suwan 1。KS23 还含有 30\%的温带玉米血 缘。经用双列杂交、顶交、优良适应种质 $\times$ 外来种 质法等分析, 合成的 10 个 Comp, Syn 及从杂交种 $\mathrm{F}_{2}$

表 2 不同群体选育出的自交系名称及其应用

\begin{tabular}{lcc}
\hline 复合(综合)种名称 & 自交系名称 & 自交系应用 \\
\hline Suwan1 硬粒型 & Ki1-Ki19, Ki22-29, Ki31-34, Ki46 & SW2301, 2602, 3101, 3501, 3851, KESX1 \\
Suwan2 硬粒型 & Ki40 & - \\
Suwan3 硬粒型 & Ki35-Ki38, Ki43 & SW3502, 3503,KSX4801 \\
Suwan5 硬粒型 & $?$ & KSX4802 \\
KS24 硬粒型 & - & - \\
Caripeno DMR 马齿型 & Ki20, Ki30 & SW2602 \\
KS6 马齿型 & Ki44, Ki47 & SW3504, 3601, 4452, KSX4804 \\
KS23 马齿型 & Ki23-F4-S7-1-4, S7-1-5, S7-6-4, S7-7-2 & KSX4803 \\
KS27 马齿型 & - & - \\
KS28 马齿型 & - & SW3101, 3501, 3502, 3503, 3504, 3601, 3851 \\
Pac9F2 马齿型 & Ki21, Ki45 & SW4452, KSX4701, 4803, 4804, KESX1, KESX3 \\
Pn3013F2 马齿型 & Kei0102 &
\end{tabular}



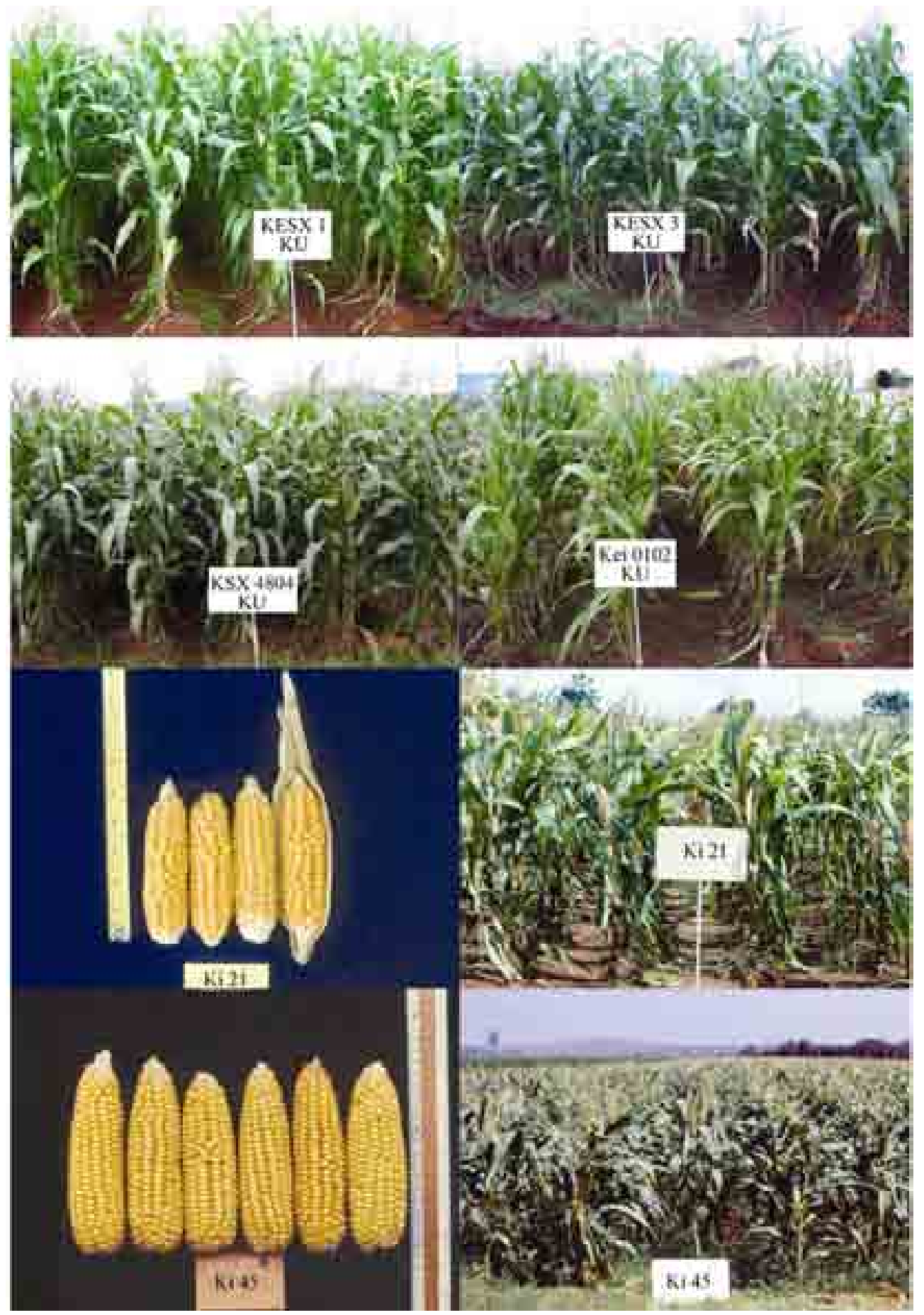

图 2 KESX1、KESX3、KSX4804 杂交种与 Kei0102、Ki21、Ki45 的特征图

选育的 Ki21(含 Ki45)、Kei0102 等可划分为 Suwan 1(含 Suwan 2、3 与 KS24), Caripeno DMR(含 KS6、 27), KS23(含 Suwan 5), Ki21(含 Ki45), Kei0102 及 CIMMYT-Pop28, KS28 等共 6 个优势群, 形成 5 个 优势模式, 即 Suwan 1×Caripeno DMR, (Suwan 1,
Caripeno DMR) $\times$ Ki21, Ki23×Ki24 (Suwan 1), (Suwan 1, 5, KS6, KS23, Ki21) $\times$ Kei0102, Suwan $1 \times$ Pop2 $8^{[4 \sim 6]}$ 。国际上提供过 Suwan 1 分别与 Reid YD、YZ No.5、Tuxpeno、Amarillo Cristalino、Late YD、Veracruz181 等构成的优势模式 ${ }^{[7 \sim 12]}$ 。 

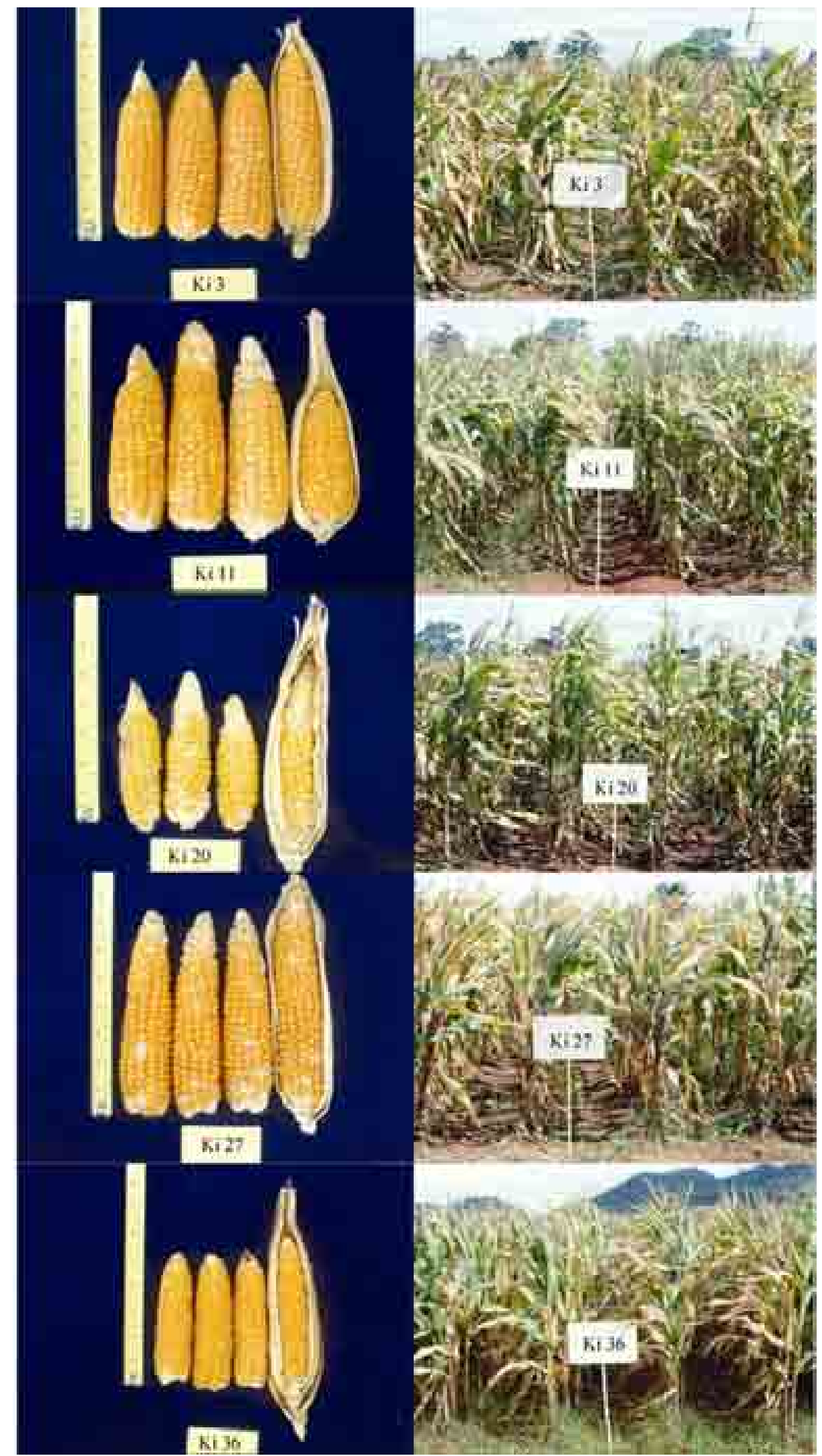

$\mathrm{Ki} 36$

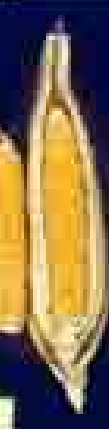




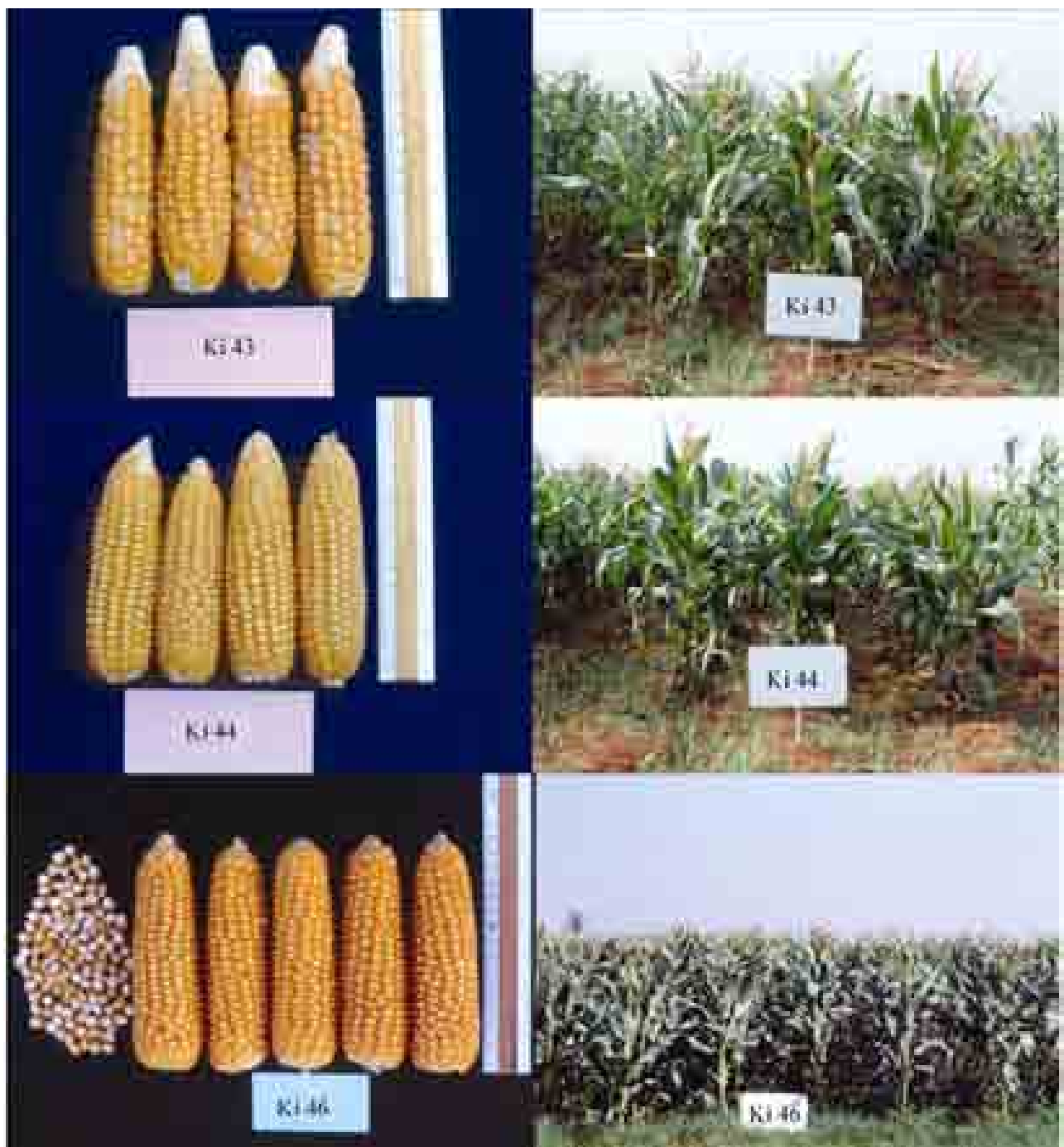

图 3 从合成复(综)合种选育的自交系特征图

\section{3 讨 论}

3.1 关于合成复合(综合)种的取材适度多样化

Suwan(KS)Comp, Syn 遗传组成是适度多样化 的。Suwan 1 包括来自加勒比的 4 个群(Argentino, Canilla, Criollo 与 Tuson) 16 份材料, 来自墨西哥和中 美洲的 4 个群(Tuxpeno, Salvadoreno, Argentino 与 Criollo)的 6 份材料, 来自南美洲的 5 个群(N.Catata, Cuban YD,Argentino,Criollo 与 Tuson)的 5 份材料, 来自印度的 4 个群(Caribbean, Tuxpeno, India 与 USA) 的 5 份材料及来自其他地区的 4 个群(Tuxpeno, Caribbean, USA 与 Phil)的 6 份材料等共 38 份, 其中 有适应力强, 抗生物与非生物胁迫的适应 OPVs, 也
有大量外来的抗霜霉、茎腐、南方锈、小斑病，丰 产稳产性好的优良 OPVs; KS23 的 26 份组成系中, 有来自温带(美国、欧洲等)、亚热带、热带(尼日利 亚、古巴、墨西哥、CIMMYT、阿根廷等)。包括马 齿类型 6 个, 半马齿 4 个, 半硬粒、硬粒类型 16 个。 总的说来, 取材适度多样化, 组装进大量玉米群 (Group)、类型(Type)与种族(Race)，其规模、严格选 择标准、预期达到目标及最终提供的成果，可与美 国 BSSS、BSCB1，中南美 Eto、THG，与中国中综、 辽旅、辽综、吉综 $A 、 B 、 W B M$ 等 $^{[7 \sim 10]}$ 相謧美。

从全国种植地域面积、跨越生态区多少，选育 的自交系配合力、主要农艺性状表现，参配组合，增 产幅度与品质优良程度, 产生的社会经济生态效益 
等方面考虑, Suwan 1 为最佳, 作为独立优势群, 在 国内国外已形成多个优势模式，对玉米遗传育种发 展有重要科学意义, 对玉米育种生产有重要经济与应 用价值, 通过几年的积极努力, 已经找到并合成与 Suwan 1(或 KS24)相对应的优势群(KS23), KS24 与 KS23 是一对新的优势模式，可以与 BSSS 同 BSCB1, Tuxpeno 同 Eto, Ta-Hu 同 Pion A(Improved Reid YD), Euro EF 同 B13(Improved BSSS)相比拟 ${ }^{[7 \sim 9,13 \sim 21]}$ 。

3.2 关于合成复合(综合)种间育种素材的亲缘关系 与优势群模式的创新

10 个 Comp, Syn 中, 其中 7 个(含 Suwan 1)均组 装了泰国生产用 OPVs(Tequisate GYF 等)或以 Suwan 1 参与, 另 3 个则组装了泰国生产用 OPVs(Caripeno DMR Comp, Caribbean Flint Comp 等)。KS6 为 Non-Suwan 1 血缘, 但组装了 Suwan 1、Amarillo Dentado、Caripeno 等 3 份与 Suwan 1 遗传组成相关 的材料。KS28 为 Non-Suwan 1 血缘, 其中 50\%为 Tuxpeno 血缘, 它也组装了 Ki22一来自 Suwan 1(s)C6 与 Ki45, Tuxpeno-1,Caripeno 等 4 份与 Suwan 1 遗传组成相关材料。因此可以说 KS6、KS28 或多 或少具有 Suwan 1 血缘, 而 Suwan 2、KS24、Suwan 3 与 Suwan 5 则是 Suwan 1 的衍生 Comp 或 Syn。 Caripeno DMR、KS23 与 KS27 均为 Non-Suwan 1 血 缘。仔细分析可知, Caripeno DMR 遗传组成 Caribbean,Tuxpeno 与 Phil 3, Phil 5 都是 Suwan 1 合 成育种素材; KS27 为 7 份 Caripeno DMR 不同轮次 群体, 分离出 $\mathrm{S} 1$ 系后, 以测交方案合成, 血缘仍与 Suwan 1 有相同点; KS23 合成育种素材中 Ki20、Ki30 来自 Caripeno DMR, 血缘仍与 Suwan 1 有相同点, 不过合成前曾将 26 份合成系与 Suwan 1 Comp 作顶 交, 均呈现高度配合力, 似乎上述的一些相同点不 足于影响到 KS23, KS24 这对相互轮选 Syn 的相对独 立性地位。如上所述, 10 个 Comp, Syn 可划分为 6 个优势群，形成 5 个优势模式。

就国际上对优势群研究现状来看, Suwan 1 和 KS24、Suwan 2, Suwan 3 的遗传组成至少包括有如 下优势群：Tuxpeno, Tuxpeno1, Antigua, Caribbean Flint, Cuban Flint, Veracruz, Tuson, Cateto, Caripeno, Tuxantigua, Usantigua, Dentado Amalillo 等。它们作 为新的独立优势群, 可与 Reid YD, YZ No.5, Tux- peno, Amarillo Cristlino, Late YD, Veracruz 181 等优 势群组成新优势模式。最近有些学者在探讨更新的 优势群(Tuxpeno×Reid YD, 形成 Tuxreid; Suwan 1× Lancster SC, 形成 Suwlanc), 组成崭新的优势模式 : Tuxreid $\times$ Suwlanc。这也是实施和验证 Hallauer 等 ${ }^{[10 ~ 14,22]}$ 提出的 BSSS-Tuxpeno $\times$ Non BSSS-Non Tuxpeno 通用 模式的一种途径。

\section{3 关于十个合成复合(综合)种给杂交优势育种的} 启示

从泰国玉米育种生产历程看, 1932 1982 年为 OPVs 时期, 1982 年开始, 以 SW2301 单交种发放为 标志, 进入单(三)交种时期, 此后相继发放了 16 个 单(三)交种。育种生产经历了两个时期，不像美国、 中国及其他一些国家那样经历典型的 4 个时期 ${ }^{[10,13]}$ 。 但 Comp, Syn 时间较长, 目前仍占有一定面积(约 $15 \% \sim 18 \%)$ 。通过 5 次规模化的引进新种质, 并把 适应品种与适应外来种质合成近 50 个轮选基础群 体，所以，已掌握的合成育种素材遗传基础丰富， 近期还不断加入新材料, 又进一步拓宽了遗传基础, 有足够的遗传变异, 有利基因频率高, 加性遗传方 差均较显性遗传方差大, 不仅可以提高群体自身的 产量, 而且可提高自交系产种量, 配合力水平及其 杂交组合优势水平。遗传产量与实际产量差距小, 增产潜力足够使用一段时间。近期 NCSRC/FS KU 选育成的优良系约 $80 \%$ 来自 Comp, Syn, 20\%来自(优 良)杂交种(组合) $F_{2}$ 大群体。Sprague 等 ${ }^{[7,8]}$ 在总结各 种选择改良法时, 得出过已有的方法每轮遗传增益 大体上都处在 3\% 4\%之间，NCSRC/FS KU 采用 S1 选择法, 将群体改良与选系结合进行, 简捷可行的 手段, 有效地推动了杂交玉米的发展, 也为实施长 期发展综合种规划，按外来种质性状划分与归纳类 群(Group)、利用国际种质库玉米种族于杂交优势育 种的长远目标，奠定了坚实的理论、技术方法与物 质基础 ${ }^{[3,5]}$ 。

\section{参考文献(References):}

[1] Sutat S, Sujin J, Vasal SK. Suwan-1: Maize from Thailand to the World. CIMMYT, Mexico, D.F. 1993.

[2] Sriwatanapongse S. Farm Suwan and corn improvement in Thailand. In: Proc Int Plant Genet Breed, Protect Acad Conf. Pakchong, Thailand, 2005, 1-5. 
[3] Jampatong S. Insight of maize breeding research of Kasetsart University. In: Proc Int Plant Genet Breed, Protect Acad Conf, Pakchong, Thailand, 2005, 12-14.

[4] Jampatong S, Rungchang P. Maize production and research for genetic improvement in Thailand. Hereditas, 2000, 22(6): 423-429.

[5] Jampatong S, Aekatasanawan C, Balla C, Chulchoho N, Sangkaeo K. An overview of population development for supporting hybrid maize breeding program. In: Proc 13th NCSRC Conf. Uben Rachathani, Thailand, 2001, 220.

[6] Chokechai A. Hybrid maize technology for rural development in Thailand. In: Proc Int Conf (The year 2000: Tech. for Rural Development). Bangkok, Thailand, 2000, 25-26.

[7] Sprague GF. Corn and corn improvement. Madison: American Society of Agronomy, 1977.

[8] Hallauer AR. 中国农业科学院作物育种栽培研究所. 玉 米轮回选择的理论与实践. 北京: 中国农业出版社, 1989.

[9] 刘纪麟. 玉米育种学. 北京: 中国农业出版社, 2002.

[10] 张世煌, 田清震, 李新海, 李明顺, 谢传晓. 玉米种质 改良与相关理论研究进展. 玉米科学, 2006, 14(1): 1-6.

[11] Hallauer AR. Introgression of elite subtropical and tropical germplasm with US Corn Belt germplasm. North Central Regional Corn Breeding Meeting, America, 2003.

[12] 陈泽辉. 玉米 Tuxpeno 和 Suwan 种质的改良研究. 2009 年全国玉米遗传育种研讨会“热带亚热带玉米种质改良
及利用” 论文摘要集. 南宁: 广西农业科学院, 2009.

[13] 曾孟潜. 我国玉米杂交优势利用回顾与未来育种改进遗 传性状的前瞻. 中国农业科技导报, 2009, 11(S2): 1-6.

[14] 曾孟潜, 吉海莲, 李九云, 三森·健萉粀. 玉米杂种优势 群及其杂交利用模式概念的形成与发展. 华北农学报, 2007, 22(6): 30-37.

[15] 曾三省, 任蓕, 刘新芝. 黄早四在我国玉米育种和生产 中的重要地位. 玉米科学, 1996, 4(1): 1-6.

[16] 李遂生. 玉米"黄早四"的选育过程及其应用. 北京农业 科学, 1997, 15(1): 19-21.

[17] 段运平, 陈卫国, 李明顺, 李新海, 刘雪, 田清震, 白丽, 张世煌. 利用 SSR 标记分析 27 个玉米群体的遗传关系. 中国农业科学, 2006, 39(6): 1102-1113.

[18] Sinobas J, Monteagudo I. Heterotic patterns among U.S. corn belt and Spanish maize populations. Maydica, 1996, 41: 143-148.

[19] Everelt LA, Eta-Ndu JT, Ndioro M, Walker P. Combining ability among source populations for tropical mid-altitude maize inbreds. Maydica, 1995, 40(2): 165-171.

[20] Vasal SK, Dhillon BS, Srinivasan G, Mclean SD, Zhang SH, Gonzalez F. Breeding intersynthetic hybrids to exploit heterosis in maize. Maydica, 1994, 39(3): 183-186.

[21] 田清震, 张世煌, 李新海, 李明顺, 谢传晓. 玉米育种 发展动态. 玉米科学, 2007, 15(1): 24-26.

[22] 孙友位. 利用 SSR 标记分析玉米自交系的遗传多样性 [学位论文]. 北京: 中国农业科学院作物科学研究所, 2007.

\section{•综合信息・}

\section{1 年第 12 期《遗传》封面说明}

多效基因指同时控制多个性状的基因, 当其突变后会引起多个性状的表型改变。研究表明, 植物中存在许多多效基 因, 它们在植物营养生长和生殖发育的调控中起着关键作用。本研究分析了一个新的水稻多效基因 DDF1, 它同时控制 水稻的营养生长和生殖发育。该基因突变后, 水稻营养器官与花器官的发育均发生明显异常。与野生型相比, 其根、茎、 叶等均变得明显细小; 穗变短, 一、二次枝梗和小穗数目明显减少, 小花内外稃不能闭合, 浆片转变为稃片, 雄蕊部分 或完全转化为雌芯, 雌荵畸形, 完全不育。通过连锁分析, 李生平等将 DDF1 定位到水稻第 6 号染色体上一个 $165 \mathrm{~kb}$ 的 区间内。根据水稻基因组注释, 该区间内存在 2 个 F-box 基因。已知 F-box 蛋白参与调控植物多种生长发育过程, 如光 形态建成、自交不亲和、生物钟节律、花发育、叶片发育等。这 2 个 F-box 基因有可能是 DDF1 的候选基因。该结果 为克隆 $D D F 1$ 基因奠定了基础。封面图片显示的是 $d d f 1$ 突变体小穗的形态特征。ddf1 突变体小花内外稃不能闭合, 浆 片转变为稃片, 雄芯转化为雌芯, 雌芯畸形。详见本期第 1374 1379 页李生平, 段远霖, 陈志伟, 官华忠, 王传蕾, 郑磊 否, 周元昌, 吴为人的“水稻生长发育多效基因 $D D F 1$ 的遗传分析与基因定位”一文。 